\title{
Magnetic-field-induced singularities in spin-dependent tunneling through InAs quantum dots
}

\author{
I. Hapke-Wurst, ${ }^{1}$ U. Zeitler, ${ }^{1}$ H. Frahm, ${ }^{2}$ A. G. M. Jansen, ${ }^{3}$ R. J. Haug, ${ }^{1}$ and K. Pierz ${ }^{4}$ \\ ${ }^{1}$ Institut für Festkörperphysik, Universität Hannover, Appelstraße 2, D-30167 Hannover, Germany \\ ${ }^{2}$ Institut für Theoretische Physik, Universität Hannover, Appelstraße 2, D-30167 Hannover, Germany \\ ${ }^{3}$ Grenoble High Magnetic Field Laboratory, MPIF-CNRS, Boite Postale 166, F-38042 Grenoble Cedex 09, France \\ ${ }^{4}$ Physikalisch-Technische Bundesanstalt Braunschweig, Bundesallee 100, D-38116 Braunschweig, Germany
}

(Received 14 August 2000)

\begin{abstract}
Current steps attributed to resonant tunneling through individual InAs quantum dots embedded in a GaAsAlAs-GaAs tunneling device are investigated experimentally in magnetic fields up to $28 \mathrm{~T}$. The steps evolve into strongly enhanced current peaks in high fields. This can be understood as a field-induced Fermi-edge singularity due to the Coulomb interaction between the tunneling electron on the quantum dot and the partly spin-polarized Fermi sea in the Landau quantized three-dimensional emitter.
\end{abstract}

The interaction of the Fermi sea of a metallic system with a local potential can lead to strong singularities close to the Fermi edge. Such effects have been predicted more than 30 years ago for the $\mathrm{x}$-ray absorption and emission of metals ${ }^{1}$ and observed subsequently. ${ }^{2}$ Similar singularities as a consequence of many-body effects are also known from the luminescence of quantum wells. ${ }^{3}$ Matveev and Larkin predicted interaction-induced singularities in the tunneling current via a localized state, ${ }^{4}$ which were measured experimentally in several resonant tunneling experiments ${ }^{5-7}$ from twodimensional electrodes through a zero-dimensional system.

Here we report on singularities observed in the resonant tunneling from highly doped three-dimensional (3D) GaAs electrodes through an InAs quantum dot (QD) embedded in an AlAs barrier. These Fermi-edge singularities (FES) show a considerable magnetic-field dependence and a strong enhancement in high magnetic fields where the 3D electrons occupy the lowest Landau level in the emitter. We observe an asymmetry in the enhancement for electrons of different spins with an extremely strong FES for electrons carrying the majority spin of the emitter. The experimental observations are explained by a theoretical model taking into account the electrostatic potential experienced by the conduction electrons in the emitter due to the charged QD. We will show that the partial spin polarization of the emitter causes extreme values of the edge exponent $\gamma$ not observed until present and going beyond the standard theory valid for $\gamma \ll 1.4$

The active part of our samples are self-organized InAs QD's with 3-4 nm height and 10-15 nm diameter embedded in the middle of a 10-nm-thick AlAs barrier and sandwiched between two 3D electrodes. They consist of a $15-\mathrm{nm}$ undoped GaAs spacer layer and a GaAs buffer with graded doping. A typical InAs dot is sketched in inset (a) of Fig. 1, the vertical band structure across a dot is schematically shown in inset (b).

Current voltage $(I-V)$ characteristics were measured in large area vertical diodes $\left(40 \times 40 \mu \mathrm{m}^{2}\right)$ patterned on the wafer. In Fig. 1 we show a part of a typical $I-V$ curve with several discrete steps. We have demonstrated previously that such steps can be assigned to single electron tunneling from
3D electrodes through individual InAs QD's (Ref. 8) consistent with other resonant tunneling experiments through selforganized InAs QD's. ${ }^{9}$

For the positive bias voltages shown in Fig. 1 the electrons tunnel from the bottom electrode into the base of an InAs QD and leave the dot via the top. The tunneling current is mainly determined by the tunneling rate through the effectively thicker barrier below the dot (single electron tunneling regime). A step in the current occurs at bias voltages where the energy level of a dot, $E_{D}$, coincides with the Fermi level of the emitter, $E_{F}$.

In the following we will concentrate on the step labeled (*) in Fig. 1. Other steps in the same structure as well as steps observed in the $I-V$ characteristics of other structures show a very similar behavior.

After the step edge a slight overshoot in the tunneling current occurs consistent with other tunneling experiments

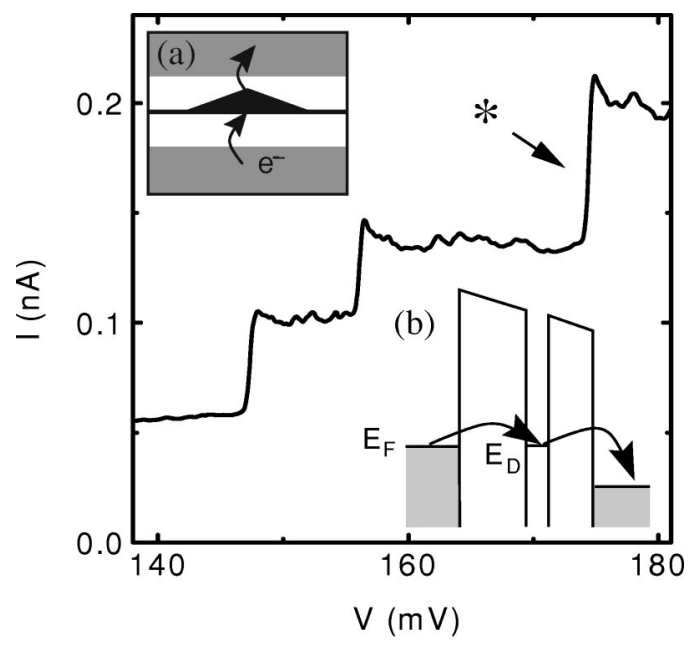

FIG. 1. Typical steps in the $I-V$ characteristics of a GaAs-AlAsGaAs tunneling diode containing InAs QD's at $T=500 \mathrm{mK}$. The (*) marks the current step due to single electon tunneling through one individual InAs QD which is analyzed in detail. Insets: (a) Principle sample structure of an InAs QD (black) embedded in an AlAs-barrier (white) between two GaAs-electrodes (grey). The arrows mark the tunneling direction of the electrons. (b) Schematic profile of the band structure at positive bias. 

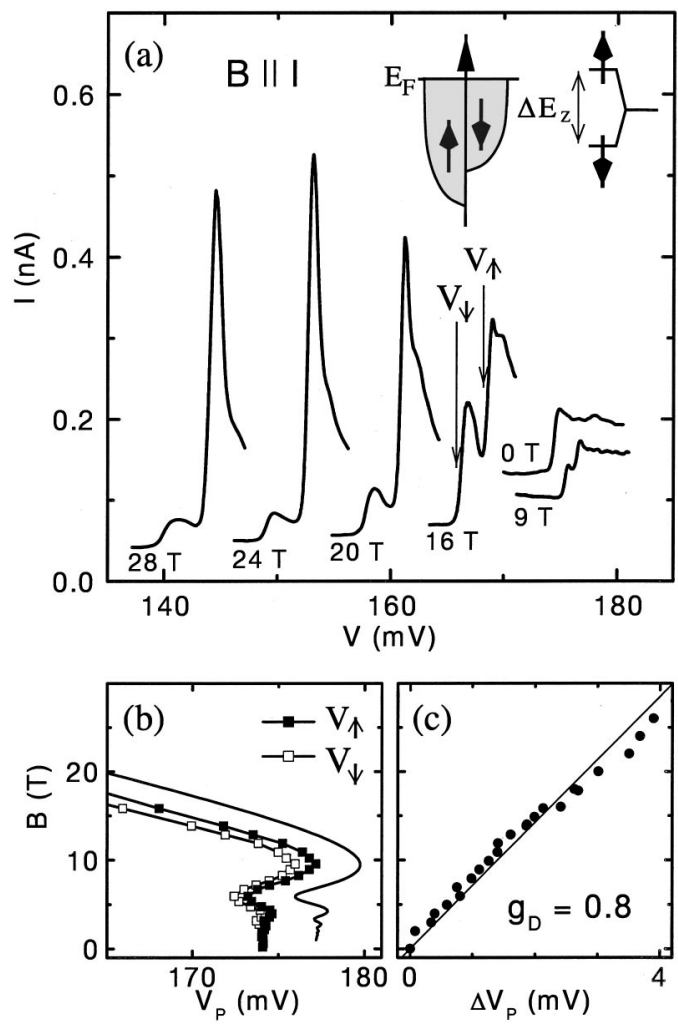

FIG. 2. (a) $I-V$ characteristics of step (*) at $T=500 \mathrm{mK}$ in various magnetic fields up to $28 \mathrm{~T}$. The inset sketches the partial spin polarization in the emitter and the spin splitting of the dot level in a magnetic field. (b) Onset voltages for the two spin-split current steps as a function of $B$ compared to the expected behavior for $E_{F}=13.6 \mathrm{meV}$ and $\Gamma=1.3 \mathrm{meV}$ (solid line, shifted to the right for clarity). (c) Measured Zeeman splitting between the two current steps.

through a localized impurity ${ }^{5}$ or through InAs dots. ${ }^{7}$ This effect is caused by the Coulomb interaction between a localized electron on the dot and the electrons at the Fermi edge of the emitter. The decrease of the current $I(V)$ towards higher voltages $V>V_{0}$ follows a power law $I \propto\left(V-V_{0}\right)^{-\gamma}$ (Ref. 5) ( $V_{0}$ is the voltage at the step edge) with an edge exponent $\gamma=0.02 \pm 0.01$.

The evolution of step (*) in a magnetic field applied parallel to the current direction is shown in Fig. 2(a). The step develops into two separate peaks with onset voltages marked as $V_{\downarrow}$ and $V_{\uparrow}$. The Landau quantization of the emitter leads to an oscillation of $V_{\downarrow}$ and $V_{\uparrow}$ and a shift to smaller voltages as a function of magnetic field, see Fig. 2(b). This reflects the magneto-quantum-oscillation of the Fermi energy in the emitter. ${ }^{12,13}$ From the period and the amplitude of the oscillation we can extract a Fermi energy (at $B=0) E_{0}=13.6$ $\mathrm{meV}$ and a Landau level broadening $\Gamma=1.3 \mathrm{meV}$ in the $3 \mathrm{D}$ emitter. The measured $E_{0}=13.6 \mathrm{meV}$ agrees well with the expected electron concentration at the barrier derived from the doping profile in the electrodes.

For $B>6 \mathrm{~T}$ only the lowest Landau level remains occupied. With a level broadening $\Gamma=1.3 \mathrm{meV}$ the Fermi level $E_{F}$ for $15 \mathrm{~T}<B<30 \mathrm{~T}$ is within less than $2 \mathrm{meV}$ pinned to the bottom of the lowest Landau band, $E_{L}=\hbar \omega_{c} / 2$. As a consequence the onset voltage shifts to lower values as $\alpha e \Delta V \approx-\hbar \omega_{c} / 2$ with $\alpha=0.34$. The diamagnetic shift of the

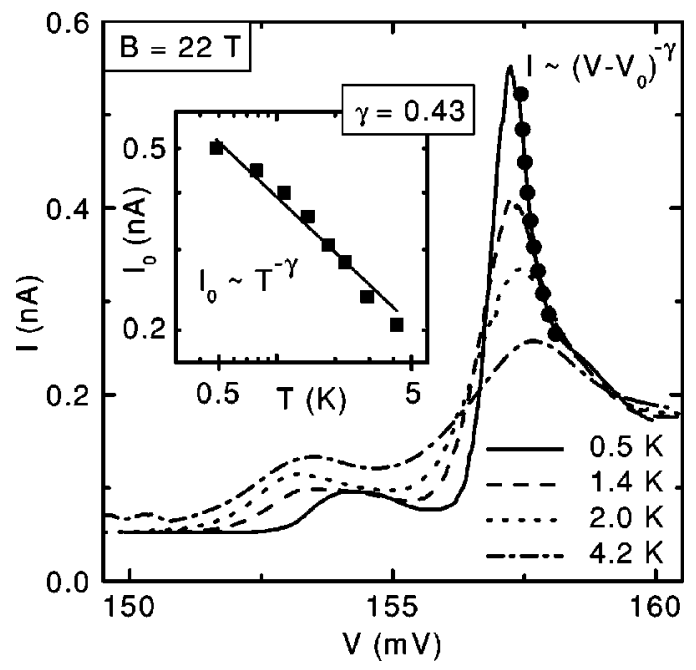

FIG. 3. $I-V$ characteristics at $B=22 \mathrm{~T}$ for different temperatures. The dots represent the shape of a FES, $I \propto\left(V-V_{0}\right)^{-\gamma}$, with an edge exponent $\gamma=0.43$. The inset shows the peak height as a function of temperature with the solid line fitting the peak current $I_{0}$ by a power law $I_{0} \propto T^{-\gamma}$.

energy level in the dot can be neglected compared to this shift of the Fermi energy in the emitter. For the dot investigated in Ref. 8 with $r_{0}=3.7 \mathrm{~nm}$ the diamagnetic shift at $30 \mathrm{~T}$ is $\Delta E_{D}=3.5 \mathrm{meV}$ negligible compared to $E_{L}=26 \mathrm{meV}$.

The two distinct steps with onset voltages $V_{\downarrow}$ and $V_{\uparrow}$ originate from the spin-splitting of the energy level $E_{D}$ in the dot. Their distance $\Delta V_{p}$ is given by the Zeeman splitting $\Delta E_{z}=g_{D} \mu_{B} B=\alpha e \Delta V_{p}$ with an energy-to-voltage conversion factor $\alpha=0.34 .{ }^{10}$ As shown in Fig. 2(c) $\Delta V_{p}$ is indeed linear in $B$, with a Landé factor $g_{D}=0.8$ in agreement with other experiments on InAs dots. ${ }^{11}$

For low magnetic fields $[B \leqslant 9 \mathrm{~T}$ in our case, see the graph for $B=9 \mathrm{~T}$ in Fig. 2(a)] the size of the steps is very similar for both spins and about half of the size at zero field. Also the slight overshoot in the current as the signature of a Fermi-edge singularity is similar for both spin orientations and comparable to the zero-field case with an edge exponent $\gamma<0.05$ for all magnetic fields $B<10 \mathrm{~T}$.

The form of the current steps changes drastically in high magnetic fields where only the lowest Landau level of the emitter remains occupied. In particular, the second current step at higher voltage evolves into a strongly enhanced peak with a peak current of one order of magnitude higher compared to the zero-field case.

Following Ref. 11 we assume that $g_{D}$ is positive whereas the Landé factor in bulk GaAs is negative. This assumption is verified by the fact that the energetically lower lying state (first peak in Fig. 3) is thermally occupied at higher temperatures and can therefore be identified with the minority spin in the emitter. The strongly enhanced current peak at higher energies is due to tunneling through the spin state corresponding to the majority spin (spin up) in the emitter. The resulting spin configuration is sketched in the inset of Fig. 2(a) and will also be confirmed below by our theoretical results.

The shape of this current peak can be described by a steep ascent and a more moderate decrease of the current towards higher voltages. Down to temperatures $T<100 \mathrm{mK}$ the 


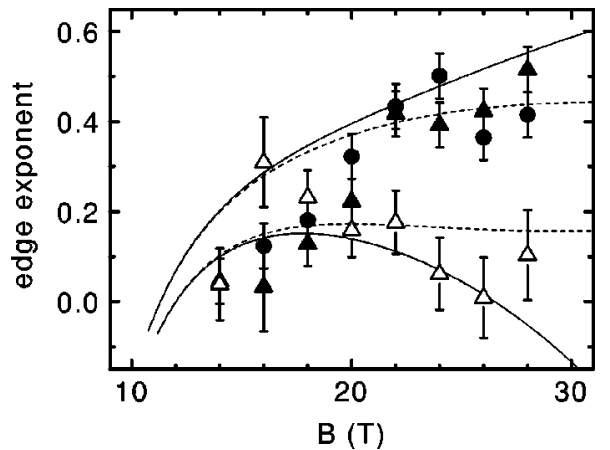

FIG. 4. Experimental edge exponents $\gamma$ extracted from the temperature dependence of the peak height (circles) and from fitting the slope (triangles) compared to our theoretical predictions. The majority spin in the emitter is shown with filled symbols, open symbols correspond to the minority spin. The solid lines represent the theoretical prediction without level broadening, for the dashed curves a level broadening $\Gamma=1.3 \mathrm{meV}$ was included in the theory.

steepness of the ascent is only limited by thermal broadening. The decrease of the current for $V>V_{0}$ is again described with the characteristic behavior for a Fermi-edge singularity, $I \propto\left(V-V_{0}\right)^{-\gamma}$, where $V_{0}$ here is the voltage at the maximum peak current. However, along with the drastic increase of the peak current the edge exponent $\gamma$ increases dramatically reaching a value $\gamma>0.5$ for the highest fields.

A different way to visualize the signature of a FES is a temperature-dependent experiment. As an example we have plotted the $I-V$ curve at $B=22 \mathrm{~T}$ for different temperatures in Fig. 3. As shown in the inset the peak maximum $I_{0}$ for the spin-up electrons decreases according to a power law $I_{0}$ $\propto T^{-\gamma}$ with an edge exponent $\gamma=0.43 \pm 0.05$. Such a strong temperature dependence is characteristic for a FES and allows us to exclude that pure density of states effects in the 3D emitter are responsible for the current peaks in high magnetic fields. As shown in Fig. 3 an edge exponent $\gamma=0.43$ also fits within experimental accuracy the observed decrease of the current for $V>V_{0}$.

It is not possible to extract the edge exponent for the minority spin directly from temperature-dependent experiments. At high magnetic fields the observed increase of the current with increasing temperature is mainly caused by an additional thermal population of the minority spin in the emitter. The general form of the curve is merely affected by temperature. Therefore, the edge exponent can only be gained from fitting the shape of the current peaks.

A compilation of the edge exponents $\gamma$ for various magnetic fields and both spin orientations is shown in Fig. 4. For the data related to the majority spin two independent methods were used to extract $\gamma$. For the minority spin only fitting of the shape of the $I-V$ curves was used.

For a theoretical description of these effects we consider a $3 \mathrm{D}$ electron gas in the half space $z<0$. In a sufficiently strong magnetic field $B \| \hat{z}$ all electrons are in the lowest Landau level. This defines a set of one-dimensional channels with momentum $\hbar k$ perpendicular to the boundary. This situation is different from the cases considered for scattering off point defects as in Refs. 1 and 4 or for a 2D electron gas where the current is carried by edge states. ${ }^{14}$ The singleparticle wave functions in channel $m \geqslant 0$ are $\psi_{m}(\rho, \phi) \sin k z$ with $\psi_{m}(\rho, \phi) \propto \rho^{m} \exp \left(-i m \phi-\rho^{2} / 4 l_{0}^{2}\right)$. In the experiments the magnetic length $l_{0}=\sqrt{\hbar / e B}\left(l_{0}=5.6 \mathrm{~nm}\right.$ at $\left.20 \mathrm{~T}\right)$ is comparable to the lateral size of the QD $2 r_{0} \approx 7 \mathrm{~nm}$. Hence the effect of the electrostatic potential of a charged dot on the electrons in a given channel of the emitter decreases rapidly with $m$, and the observed FES are mainly due to the tunneling of electrons from the $m=0$ channel into the dot. Following Refs. 1 and 4 tunneling processes of spin $\sigma$ electrons from the $m=0$ state in the emitter give rise to a FES with edge exponent

$$
\gamma_{\sigma}=-\frac{2}{\pi} \delta_{0}\left(k_{F \sigma}\right)-\frac{1}{\pi^{2}} \sum_{m} \sum_{\tau=\uparrow, \downarrow}\left[\delta_{m}\left(k_{F \tau}\right)\right]^{2},
$$

where $\delta_{m}(k)$ is the Fermi phase shift experienced by the electrons in the $m$ th channel due to the potential of the quantum dot. ${ }^{15}$ From Eq. (1) the observed field dependence of the edge exponents is a consequence of the variation of the Fermi momenta for spin- $\sigma$ electrons with magnetic field and the field dependence of the effective potential in the onedimensional channels. The former can be computed from the one-dimensional density of states (DOS) of the lowest Landau band

$$
D(E, B)=\frac{e \sqrt{m^{*}}}{(2 \pi \hbar)^{2}} B\left[d\left(\epsilon_{\uparrow}\right)+d\left(\epsilon_{\downarrow}\right)\right] .
$$

Here $\epsilon_{\sigma}=E-\left(\hbar \omega_{c} \pm g^{*} \mu_{B} B\right) / 2$ is the energy of electrons with spin $\sigma$ measured from the bottom of the Landau band. $g^{*} \approx-0.33$ (Ref. 16) is the effective Lande factor of the electrons in the emitter. The DOS for the spin subbands is $d(\epsilon)=\sqrt{2} \operatorname{Re}(\epsilon+i \Gamma)^{-1 / 2}$. Without broadening, $\Gamma=0$, one has $k_{F \sigma}=\pi^{2} n l_{0}^{2}\left(1 \pm b^{3}\right)$ where $n$ is the 3D density of electrons and $b$ is the magnetic field measured in units of the field necessary for complete spin polarization of the 3D emitter. Using a Fermi energy $E_{0}=13.6 \mathrm{meV}$ and neglecting level broadening we find that only the lowest Landau level (both spin states) is occupied for $B_{1}>5.2 \mathrm{~T}$. Including level broadening changes $B_{1}$ to a slightly higher value. With the known field dependence of the Fermi energy in the quantum limit we can calculate the field for total spin polarization

$$
B_{p o l}=\left(\frac{16}{9 \xi}\right)^{1 / 3} \frac{m^{*} E_{0}}{\hbar e} \simeq 43 \mathrm{~T}
$$

with $\quad g^{*}=-0.33 \quad$ (Ref. 16) and $m^{*}=0.067 m_{0} . \quad \xi$ $=\frac{1}{2}|g *| m^{*} / m_{0}$ is the ratio between spin splitting and Landau level splitting.

To make contact with the experimental observations we have to specify the interaction of the screened charge on the QD and the conduction band electrons. A Thomas-Fermi calculation gives $U(\rho, z)=\left[2 e^{2} \exp (\kappa z) / \kappa\right)\left(d /\left(\rho^{2}+d^{2}\right)^{(3 / 2)}\right]{ }^{4}$ Here $d=5 \mathrm{~nm}$ is the width of the insulating layer and $\kappa^{-1}$ $=7 \mathrm{~nm}$ is the Debye radius. The effective potential seen by electrons in channel $m$ is $V_{m} \exp (\kappa z) / \kappa$ with $V_{m}$ $=2 e^{2} d \int d \rho^{2}\left|\psi_{m}(\rho, \phi)\right|^{2} /\left(\rho^{2}+d^{2}\right)^{(3 / 2)}$. For large $\kappa$ we obtain for the phase shift in the $m=0$ channel $\delta_{0}(k) \approx$ $-v_{0} f(B) k / \kappa$ where 


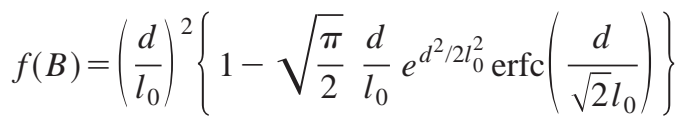

and $v_{0} \sim\left(m^{*} e^{2} / \hbar^{2} \kappa\right)(\kappa d)^{-2}$ up to a numerical factor. Similarly we obtain the integrated effect of the channels $m>0$ in Eq. (1). In Fig. 4 the resulting exponents $\gamma_{\sigma}$ obtained for $\sigma$ $=\uparrow, \downarrow$ are shown for $v_{0}=6.75$ and a broadening $\Gamma=0$ and $\Gamma=1.3 \mathrm{meV}$, respectively. The value used for $\Gamma$ reflects its realistic experimental value. $v_{0}$ is the only fit parameter.

Already the simple model with no level broadening $(\Gamma$ $=0$ ) is in good agreement with the experimentally measured edge exponents for both spin directions, especially in high magnetic fields where possible admixtures of higher Landau levels play a minor rule. Including level broadening leads to a less dramatic spin polarization in the emitter and as a consequence smears out the field dependence of $\gamma$ for the minority spin. The basic features, however, remain unchanged. In particular, the edge exponent for the minority spin retains moderate values for high magnetic fields, whereas the edge exponent related to the majority spin shows a strong field dependence with very high values in high magnetic fields.

In conclusion we have evaluated experimental data concerning magnetic-field-induced FES in resonant tunneling experiments through InAs QD's. We have shown that the interaction between a localized charge and the electrons in the Landau quantized emitter leads to dramatic Fermi phase shifts if only the lowest Landau level in the 3D emitter is occupied. This results in edge exponents $\gamma>0.5$ which were measured and described theoretically.

We would like to thank H. Marx for growing the samples, P. König for experimental support, and F. J. Ahlers for valuable discussions. Part of this work has been supported by the TMR Programme of the European Union under Contract No. ERBFMGECT950077. We acknowledge partial support from the Deutsche Forschungsgemeinschaft under Grant Nos. HA 1826/5-1 and Fr 737/3.
${ }^{1}$ G. D. Mahan, Phys. Rev. 163, 612 (1967); Many-Particle Physics (Plenum, New York, 1981); P. Noziéres and C. T. De Dominicis, Phys. Rev. 178, 1097 (1969); K. D. Schotte and U. Schotte, ibid. 182, 479 (1969).

${ }^{2}$ See, for example, T. A. Callcott, E. T. Arakawa, and D. L. Ederer, Phys. Rev. B 18, 6622 (1978), and references therein.

${ }^{3}$ M. S. Skolnik, J. M. Rorison, K. J. Nash, D. J. Mowbray, P. R. Tapster, S. J. Bass, and A. D. Pitt, Phys. Rev. Lett. 58, 2130 (1987); J. A. Brum and P. Hawrylak, Comments Condens. Matter Phys. 18, 135 (1997).

${ }^{4}$ K. A. Matveev and A. I. Larkin, Phys. Rev. B 46, 15337 (1992).

${ }^{5}$ A. K. Geim, P. C. Main, N. La Scala, Jr., L. Eaves, T. J. Foster, P. H. Beton, J. W. Sakai, F. W. Sheard, M. Henini, G. Hill, and M. A. Pate, Phys. Rev. Lett. 72, 2061 (1994).

${ }^{6}$ D. H. Cobden and B. A. Muzykantskii, Phys. Rev. Lett. 75, 4274 (1995).

${ }^{7}$ K. A. Benedict, A. S. G. Thornton, T. Ihn, P. C. Main, L. Eaves, and M. Henini, Physica B 256-258, 519 (1998).

${ }^{8}$ I. Hapke-Wurst, U. Zeitler, H. W. Schumacher, R. J. Haug, K. Pierz, and F. J. Ahlers, Semicond. Sci. Technol. 14, L41 (1999).

${ }^{9}$ I. E. Itskevich, T. Ihn, A. Thornton, M. Henini, T. J. Foster, P. Moriarty, A. Nogaret, P. H. Beton, L. Eaves, and P. C. Main, Phys. Rev. B 54, 16401 (1996); T. Suzuki, K. Nomoto, K. Taira, and I. Hase, Jpn. J. Appl. Phys., Part 1 36, 1917 (1997); M. Narihiro, G. Yusa, Y. Nakamura, T. Noda, and H. Sakaki,
Appl. Phys. Lett. 70, 105 (1997).

${ }^{10}$ The energy-to-voltage conversion factor $\alpha$ is derived from the temperature dependence of the width $\delta V$ of a current step caused by the thermal smearing of the Fermi edge in the emitter. Only a part $\alpha$ of the total voltage applied drops between the emitter and the dot, the rest of the voltage drop occurs inside the electrodes and between dot and collector.

${ }^{11}$ A. S. G. Thornton, T. Ihn, P. C. Main, L. Eaves, and M. Henini, Appl. Phys. Lett. 73, 354 (1998).

${ }^{12}$ T. Schmidt, R. J. Haug, V. I. Fal'ko, K. v. Klitzing, A. Förster, and H. Lüth, Phys. Rev. Lett. 78, 1540 (1997).

${ }^{13}$ P. C. Main, A. S. G. Thornton, R. J. A. Hill, S. T. Stoddart, T. Ihn, L. Eaves, K. A. Benedict, and M. Henini, Phys. Rev. Lett. 84, 729 (2000).

${ }^{14}$ A. V. Balatsky and S. I. Matveenko, Phys. Rev. B 52, 8676 (1995).

${ }^{15}$ In fact, one has to consider the change of this phase shift due to the increase in charge on the QD here. For the contact interaction considered here this is not essential.

${ }^{16}$ P. Pfeffer and W. Zawadzki, Phys. Rev. B 41, 1561 (1990). The electronic Landé factor in the lowest Landau level in GaAs increases linearly with magnetic field from $g^{*}=-0.44$ at $B=0$ to $g^{*}=-0.29$ at $B=30 \mathrm{~T}$. Our theory uses a constant $g^{*}$, for the best possible connection with the experiment we have chosen to use its value at $B=22 \mathrm{~T}$. 